\title{
CONSTRAINTS ON GALAXY FORMATION FROM EXTRAGALACTIC GLOBULAR CLUSTERS
}

\author{
JEAN P. BRODIE \\ Lick Observatory \\ University of California, Santa Cruz, CA 95060, U.S.A.
}

\section{Introduction}

The merger model for elliptical galaxy formation has received increasing attention since it was first suggested by Toomre \& Toomre (1972). Van den Bergh (1984) pointed out a problem with the idea that elliptical galaxies were formed by simply combining two, or more, spiral galaxies. He noted that the specific frequency $\left(\mathrm{S}_{N}\right.$, number of globular clusters per unit galaxy light) is systematically lower for spirals than for ellipticals. Schweizer (1987) suggested that globular clusters might be expected to form in the merger process, thereby alleviating or possibly eliminating the $S_{N}$ problem. Ashman \& Zepf (1992) developed this idea into a merger model for globular cluster formation with testable predictions.

We recently examined this model in the light of new HST and groundbased imaging data on the blue and red sub-populations of globular clusters in elliptical galaxies (Forbes, Brodie \& Grillmair 1997). We concluded that the merger model for globular cluster formation has serious problems, especially in explaining the characteristics of globular clusters in giant elliptical galaxies with high $S_{N}$. For example, the globular cluster systems of high $\mathrm{S}_{N}$ galaxies do not have more metal-rich globular clusters as would be expected in the merger model but instead have more metal poor ones. This means that globular cluster metallicity gradients are steeper in high $\mathrm{S}_{N}$ galaxies, contrary to the predictions of Ashman \& Zepf (1992).

We proposed a multi-phase collapse scenario, more consistent with the available evidence (see also Kissler-Patig 1997). We speculated that there are three main phases in the collapse process (which we called pre-galaxy, galaxy and disk phases) each of which may have associated globular cluster formation. The metal-poor globular clusters form at an early stage in the collapse of the protogalactic cloud, in the pre-galaxy phase. Only a small 
fraction of the available gas is converted into stars, and most of these stars are located in globular clusters which formed from the chaotic merging of dense, gravitationally bound clumps. In the second phase, after the gas cloud has undergone further collapse, field star formation is preferred over star formation in globular clusters (possibly due to more efficient cooling at higher metallicities). Both field stars and globular clusters will have essentially the same metallicity (i.e. relatively metal-rich). Thus the metal-rich globular clusters formed out of more enriched gas, roughly contemporaneously with the galaxy stars. The disk globular clusters in spirals may represent a third phase of this formation process. Tidal stripping of the globular clusters from nearby galaxies may have contributed to the globular cluster population in the outer parts of $\mathrm{cD}$ galaxies, and recent mergers may have contributed to the globular cluster population in the centers of elliptical galaxies, but we suggest that the vast majority of globular clusters in $\mathrm{gE}$ and $\mathrm{cD}$ galaxies have probably formed in situ.

Below we describe two "case studies" which illustrate how optical spectroscopy with the new generation of $10 \mathrm{~m}$-class telescopes can help constrain both globular cluster and galaxy formation models. Some of the issues that can be addressed with spectroscopic data include the following:

- Do globular clusters in other galaxies show the same abundance ratios as those in the Local Group?

- Are globular clusters in elliptical galaxies more metal rich than in spirals, as would seem to be indicated by their colors?

- What is the upper limit on $[\mathrm{Fe} / \mathrm{H}]$ ?

- Can abundances, abundance ratios, ages and kinematics identify globular clusters that formed during a merger?

Optical broad-band colors suffer from a well-known age-metallicity degeneracy and so have been of limited value in addressing these issues.

The first case study is an examination of the globular clusters in NGC 1399. The second is a spectroscopic study of the proto-globular cluster candidates in NGC 1275.

\section{The NGC 1399 Globular Cluster System}

NGC 1399 is the $\mathrm{cD}$ galaxy at the center of the Fornax cluster at a distance of 19.3 Mpc (Madore, et al. 1997). Several studies (Ostrov et al. 1993, Kissler-Patig et al. 1997, Forbes et al. 1997) have demonstrated the existence of bimodal, and possibly trimodal, globular cluster color distributions in this galaxy. Using the Keck telescope, we recently obtained $5 \AA$ resolution spectra of 18 globular clusters in NGC 1399 (Kissler-Patig et al. 1998). Figure 1 shows three representative spectra of objects in the blue, intermediate and red sub-populations. 




Figure 1. Fig. 1. - Three representative spectra of globular clusters from the blue, intermediate and red sub-populations in NGC 1399. The spectra were smoothed over 3 pixels. While $\mathrm{H}_{\beta}$ gets slightly weaker from the blue to the red object, the metal lines $(\mathrm{Mg}, \mathrm{Fe})$ become much stronger.

In general, we found no anomalies in the strengths of absorption line indices sensitive to metals (e.g. $\mathrm{Mg}, \mathrm{Fe}$ ), nor in the more age-sensitive Balmer-line indices (Figure 2). However, our sample contained two clusters for which: 1) $\mathrm{Mg}, \mathrm{Fe}$ and $\mathrm{Na}$ were stronger than in any Milky Way or M31 globular clusters. These clusters may possibly also have a $\mathrm{Mg} / \mathrm{Fe}$ over-abundance, similar to that seen in giant elliptical galaxies. Worthey et al. (1992) discussed possible explanations for the $\mathrm{Mg} / \mathrm{Fe}$ enhancement in galaxies, perhaps the most plausible of which was a variable (flatter) IMF. 2) $\mathrm{H} \beta$ and $\mathrm{H} \gamma$ (effectively the only two Balmer lines in the spectral range) were abnormally strong. A similar effect was also seen in a few M31 globular clusters (Brodie \& Huchra 1991), although at a lower metallicity. The Balmer lines are too strong to be reproduced by the spectral synthesis models (Worthey 1994, Fritze v. Alvensleben \& Burkert 1995) at any age or metallicity. A component of blue horizontal branch stars might offer at least a partial explanation. Such stars are not represented in the models 

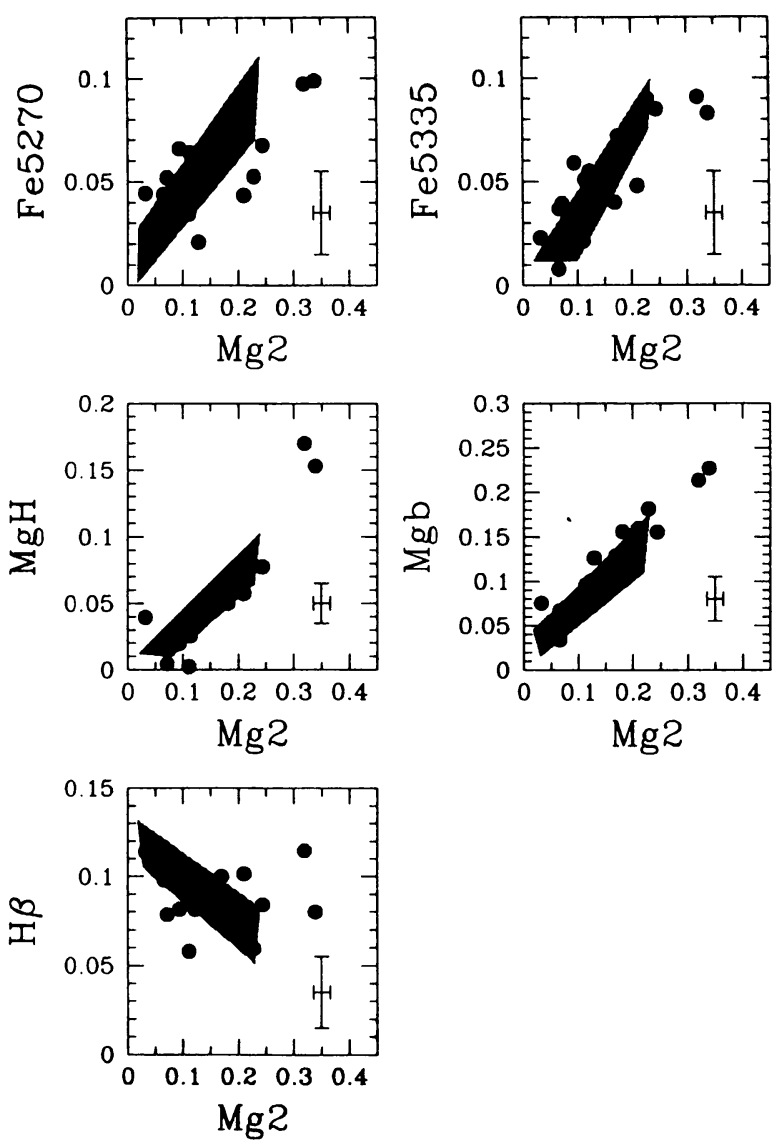

Figure 2. Fig. 2. - Mg2 versus various other indices, with the range spanned by Milky Way and M31 globular clusters shown as shaded regions (taken from Brodie \& Huchra 1990 and Burstein et al. 1984). Apart from two very metal-rich objects, all globular clusters are consistent with abundances found in the Milky Way and M31 globular clusters.

because they were not thought to be present in metal-rich clusters. However, Rich et al. (1997) recently discovered blue horizontal branches in two metal-rich globular clusters in the Milky Way, suggesting that a revision of the models may be in order.

Since the two "anomalous" NGC 1399 clusters are also very metal rich (see below), they must have formed from solar metallicity (i.e. enriched) gas, which may indicate that they are also younger than the rest. Judging by the photometric color distribution (these peculiar clusters are in the extreme red tail of the distribution), these clusters constitute less than $5 \%$ of the total globular cluster population.

We calculated mean metallicity, $[\mathrm{Fe} / \mathrm{H}]$, for all 18 clusters using the method described in Brodie \& Huchra (1990). The Mg line-strengths were first corrected for a non-linear dependence of $\mathrm{Mg}$ index strength on metal- 
licity for metallicities near and above solar, according to the models of Worthey (1994). Except for the two peculiar clusters, the range in metallicity seen in the NGC 1399 globulars is very similar to the range seen in the Milky Way and M31: The metal-poor clusters have metallicities similar to those of the Milky Way halo and the metal-rich clusters have metallicities similar to Milky Way bulge/disk globular clusters. Although we are unable to estimate age to better than a factor of two from our data, it appears that the vast majority of NGC 1399 globular clusters formed from processes similar to those which formed the Milky Way and M31 globular cluster systems, and at roughly the same epoch (i.e. they are old).

$\mathrm{V}-\mathrm{I}$ was found to correlate well with $[\mathrm{Fe} / \mathrm{H}]$ and, although individual metallicities cannot be determined to better than 0.5 dex, a mean metallicity for a globular cluster ensemble can be determined to about 0.3 dex from broad-band colors. However, the slope of the V-I vs. metallicity relation for the combination of Milky Way and NGC 1399 globular clusters was found to be twice as flat as the relation derived from Milky Way globular clusters alone (Figure 3 ). The consequence of this result is a much more homogeneous picture for extragalactic globular cluster systems. Metallicity estimates for very red globular clusters are now roughly solar, rather than very super-solar as was previously thought, and metallicity differences between galaxies' globular cluster systems are much less significant.

In summary, it appears that NGC 1399 and its globular cluster system formed at early times. The same formation processes that were responsible for forming the Milky Way globular clusters probably formed the vast majority of NGC 1399 globular clusters. Only a small percentage need have formed later from solar metallicity gas, e.g. in a merger.

\section{Proto-globular Cluster Candidates in NGC 1275}

The discovery, with HST imaging, of proto-globular cluster candidates in NGC 1275 (Holtzman et al. 1992) was regarded by many as a major success of the merger model which predicted that newly-formed clusters should be observable in currently or recently merging systems. The NGC 1275 clusters constitute an important test of globular cluster formation models. NGC 1275 is the peculiar $\mathrm{cD}$ galaxy at the center of the Perseus cluster. It shows evidence for a merger history and may indeed be undergoing a merger at present. It also has one of the largest know cooling flows. Globular cluster formation in cooling flows (Fabian et al. 1984) received some support from the photometric study of the young clusters in NGC 1275 by Richer et al. (1993).

Shown in Figure 4 are spectra of 5 proto-globular cluster candidates in NGC 1275, obtained with the Keck I telescope (Brodie et al. 1997). 


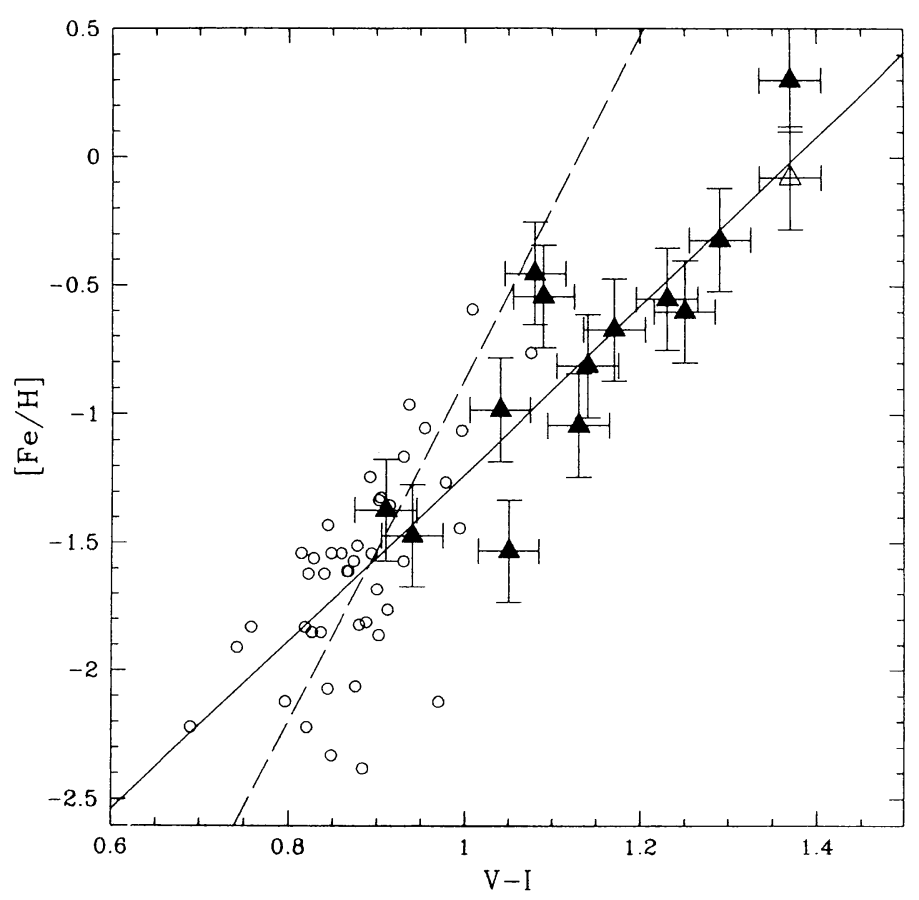

Figure 9. Fig. 3. - V-I color versus mean metallicity of the globular clusters in NGC 1399 (triangles). For cluster \#14 the metallicity derived using only the Fe absorption line index, Fe5270, is also shown (open triangle). Over-plotted as open circles are all (de-reddened) Milky Way globular clusters with $\mathrm{E}(\mathrm{B}-\mathrm{V})<0.2$ (taken from Harris 1996). The solid line is the best fit to the data, the dashed line was derived from the Milky Way sample by Kissler-Patig et al. (1997) and extrapolated to higher metallicities.

These reveal that the candidates are not HII regions, are clearly dominated by early A-type stars, and are not similar to young or intermediate age Magellanic Cloud or Milky Way open clusters.

The Balmer absorption lines were found to be too strong to be consistent with any of the standard IMF (Salpeter or Scalo), solar metallicity, Bruzual \& Charlot stellar evolutionary models at any age (Figure 5). The preliminary Bruzual \& Charlot (1997) models indicate that no appreciable increase in equivalent width can be achieved by changing the metallicity. However, a 2-3 $\mathrm{M}_{\odot} \mathrm{IMF}$, adopted to simulate a flatter IMF, reproduces the observed equivalent widths and colors and indicates an age of $\sim 500$ Myr for these objects.

The Fritze v. Alvensleben \& Kurth (1997) models can reproduce the $\mathrm{H}_{\beta}$ and $\mathrm{H}_{\gamma}$ equivalent widths without the need to invoke a non-standard IMF, although the agreement with $\mathrm{H}_{\delta}$ is less good. The best agreement is with the $350 \mathrm{Myr}$ model but the model continuum is redder than the observed spectra, even before correction for internal reddening (Brodie et al. 1997). 


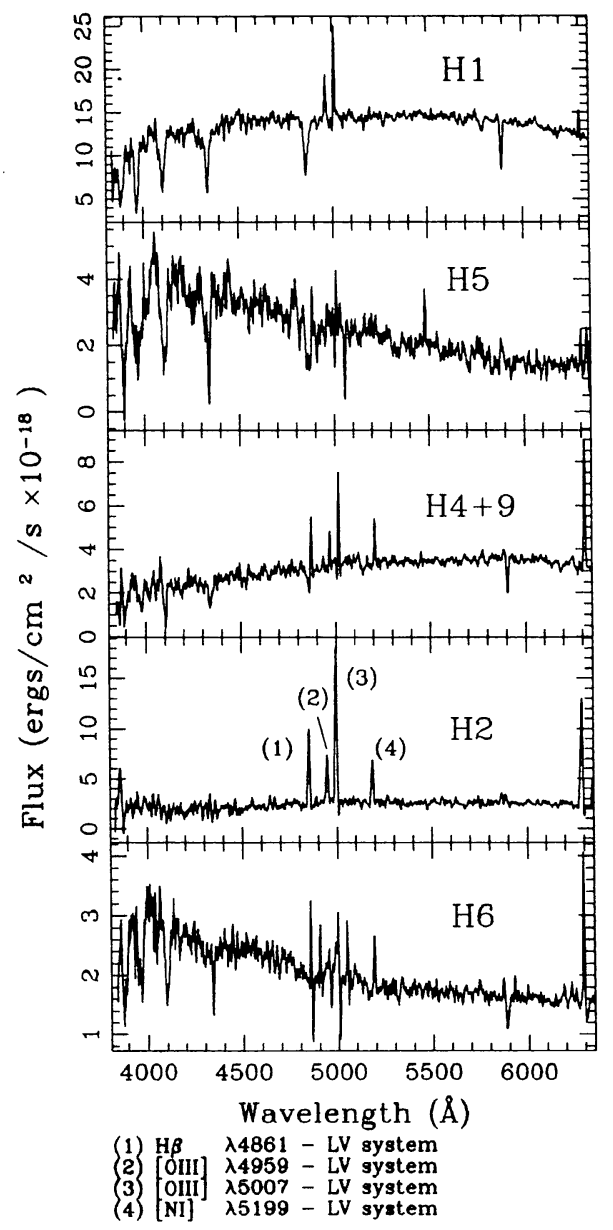

Figure 4. Fig. 4. - Keck I spectra of NGC 1275 proto-globular cluster candidates. The data have been smoothed with a box size of seven pixels and the resolution is $5.1 \AA$. The emission lines, which are residuals from imperfect subtraction of the background galaxy/gas spectrum, are marked to indicate the source of the line. LV: Residuals from the low-velocity system. HV: Residuals from the high-velocity infalling system.

The sense of the discrepancy is that the model predicts too much red light, consistent with the suggestion of a flatter cluster IMF.

Another problem with the assumption of a standard IMF for these objects is the fact that, based on their luminosities, the masses of these bright clusters are deduced to be $\sim 10^{8} M_{\odot}$. Such high mass clusters would be difficult to form, requiring surprisingly massive progenitor gas clouds, and would be very unlikely to loose sufficient mass during their evolution to bring them into the mass range of normal old globular clusters. On the other hand, a pure A-star population, for example, would have a mass 


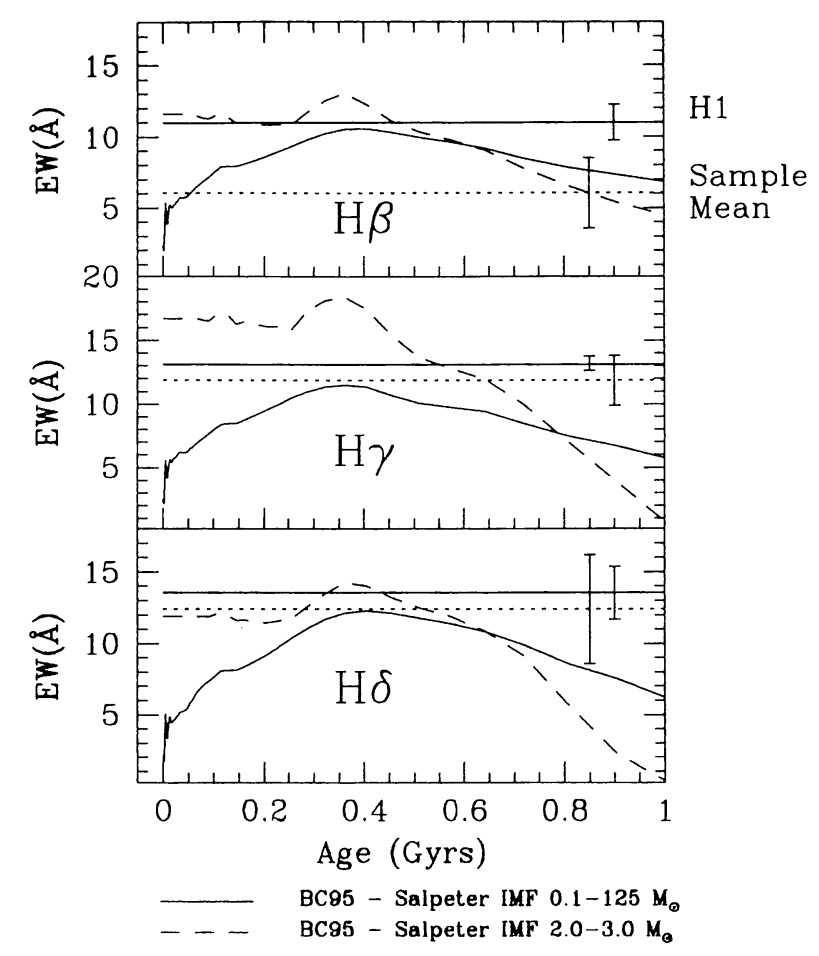

Figure 5. Fig. 5. - The time evolution of Balmer line equivalent widths for populations with different IMFs, produced by the Bruzual and Charlot models circulated in 1995. Also shown are the Balmer line equivalent widths measured for the brightest cluster, $\mathrm{H} 1$, (solid horizontal line) and the mean equivalent widths for the other clusters in the sample (dotted horizontal line). The value for $\mathrm{H} \beta$ is depressed, particularly in the lower signal-to-noise spectra, by under-subtracted $\mathrm{H} \beta$ emission in the galaxy background.

of $\sim 10^{6} M_{\odot}$ at these luminosities, and a flatter than normal IMF would produce a mass somewhere in between.

Other key properties of the proto-globular cluster candidates are their spatial distribution and their velocity dispersion. These objects are extremely centrally concentrated. The entire sample (some 60 objects) is within $8 \mathrm{kpc}$ of the nucleus and the brightest clusters are all within 2 $\mathrm{kpc}$. We find a low velocity dispersion, $\sim 200 \mathrm{~km} / \mathrm{s}$, for our sample of 5 of the brighter candidates. This is lower than estimates of the stellar central velocity dispersion, $\sim 300 \mathrm{~km} / \mathrm{s}$ (Lazareff et al. 1989).

\subsection{CONSTRAINTS ON CLUSTER ORIGINS}

The spectroscopic information allows us to set some interesting constraints on the origin of these objects. We can clearly rule out formation in a con- 
tinuous cooling flow. The spatial scale of the clusters is very much less than the cooling flow radius (by a factor of 60 for our spectroscopic sample and a factor of 20 for the entire candidate sample) and their age is very much greater than the cooling time ( $\sim 10 \mathrm{Myr})$. Moreover, a star formation rate in excess of $400 \mathrm{M}_{\odot} / \mathrm{yr}$ is deduced from the cooling flow (Allen \& Fabian 1997). This implies a steep IMF because the far UV observations (Smith et al. 1992) set severe limits on the presence of high mass stars in this galaxy. We note that, if anything, the cluster's IMF may be biased against low mass stars.

It is equally clear that these clusters did not form in widespread shocks from merging galaxies. They are centrally concentrated and, if they formed far from the center and later fell in, a high rather than a low velocity dispersion would be expected.

It appears, then, that the clusters formed in a discrete event some 500 Myr ago. This may have been induced by a merger which provided the fuel for a short-lived gas inflow episode. It is not, however, clear to what extent processes such as these may have affected the global characteristics of globular cluster systems. The resultant clusters are not distributed like old globular clusters in central $\mathrm{cD}$ galaxies which are significantly more diffuse than the galaxy light. If they do indeed have an IMF which is biased against low mass stars, they may fade very rapidly. A pure A-star population would fade away in only $\sim 10^{9} \mathrm{yr}$.

\section{Summary}

- The observational evidence fails to match merger model expectations for globular cluster formation in several respects. A multi-phase collapse scenario may be more consistent with the observed properties of extragalactic globular cluster systems.

- The vast majority of globular clusters in NGC 1399 probably formed by the same processes and at the same epoch as those responsible for forming the Milky Way globular cluster system. Only a small subset $(<5 \%)$ need have formed more recently (e.g. in a merger).

- The newly-defined $\mathrm{V}-\mathrm{I}$ versus $[\mathrm{Fe} / \mathrm{H}]$ relation suggests that metallicity differences between galaxies' globular cluster systems are much less significant than previously thought and that there are no very super-solar globular clusters.

- The proto-globular cluster candidates in NGC 1275 probably formed in a merger-induced gas infall episode some $500 \mathrm{Myr}$ ago. However, their properties are such that they may not represent formation processes that had any significant effect on the global properties of globular cluster systems. 


\section{Acknowledgments}

It is a pleasure to thank Duncan Forbes, Carl Grillmair, John Huchra, Markus Kissler-Patig, Drew Phillips and Linda Schroder who collaborated on the various parts of this work. This research was funded by HST grants GO.05990.01-94A and GO.05920.01-94A and by faculty research funds from the University of California, Santa Cruz.

\section{REFERENCES}

Ashman, K.M., \& Zepf, S.E. 1992, ApJ, 384, 50

Allen, S.W., \& Fabian, A.C. 1997, MNRAS, 286, 583

Brodie, J.P., \& Huchra, J.P. 1990, ApJ, 362, 503

Brodie, J.P., \& Huchra, J.P. 1991, ApJ, 379, 157

Brodie, J.P., Schroder, L.L., Huchra, J.P., Phillips, A.C., Kissler-Patig, M., \& Forbes, D.A. 1997, AJ submitted

Bruzual, G., \& Charlot, S. 1997, in preparation

Burstein, D., Faber, S.M., Gaskell, C.M., Krumm, N. 1984, ApJ, 287, 586

Fabian, A.C., Nulsen, P.E.J., \& Canizares, C.R. 1984, Nature, 310, 733

Forbes, D., Brodie, J.P., \& Grillmair, C. 1997, AJ, 113, 1652

Forbes, D.A., Grillmair, C.J., Williger, G.M., Elson, R.A.W., \& Brodie, J.P. 1997, MNRAS, in press

Fritze-v. Alvensleben, U. \& Burkert, A. 1995, A\&A, 300, 58

Fritze-v. Alvensleben, U. \& Kurth, O. 1997, in preparation

Harris, W.E. 1996, AJ, 112, 1487

Holtzman, J.A., et al. 1992, AJ, 103, 691

Kissler-Patig, M., Kohle, S., Hilker, M., Richtler, T., Infante, L., Quintana, H. 1997, A\&A, 319, 470

Kissler-Patig, M. 1997, PhD thesis, Sternwarte, Bonn

Kissler-Patig, M., Kohle, S., Richtler, T., et al. 1997, A\&A, 319, 470

Kissler-Patig, M., Brodie, J.P., Schroder, L.L., Forbes, D.A., Grillmair, C.G., \& Huchra, J.P. 1998, AJ in press, January issue

Lazareff, B., Castets, A., Kim, D.W., \& Jura, M. 1989, ApJ, 336, L13 Madore, B.F., Freedman, W.F. Silberman, N.A. et al. 1997, Nature, in press Nørgaard-Nielson, H.U., Goudfrooij, P., Jorgensen, H.E., \& Hansen, L. 1993, AA, 279, 61

Ostrov, P., Geisler, D., Forte, J.C. 1993, AJ, 105, 1762

Rich, R.M., Sosin, G., Djorgovski, S.G., et al. 1997, ApJ, 484, L25

Richer, H., Crabtree, D., Fabian, A.., \& Lin, D. 1993, AJ, 105, 877

Smith, E.P. et al. 1992, ApJ, 395, L49

Toomre A., Toomre J., 1972, ApJ 178, 623

Worthey, G., Faber, S.M., \& Gonzales, J.J. 1992, ApJ 398, 69

Worthey, G. 1994, ApJS, 95, 107 